\title{
Novel missense mutations in the TRPS1 transcription factor define the nuclear localization signal
}

\author{
Frank J Kaiser ${ }^{1}$, Paola Brega ${ }^{1}$, Michael L Raff ${ }^{2,3}$, Peter H Byers ${ }^{4}$, Sabina Gallati ${ }^{5}$, Teresa Taylor \\ Kay $^{6}$, Salomé de Almeida ${ }^{6}$, Bernhard Horsthemke ${ }^{1}$ and Hermann-Josef Lüdecke*,1
}

\begin{abstract}
${ }^{1}$ Institut für Humangenetik, Universitätsklinikum, Hufelandstr. 55, Essen D-45122, Germany; ${ }^{2}$ Division of Genetics and Development, Children's Hospital and Regional Medical Center, University of Washington, Box 357720, Seattle, WA 98195, USA; ${ }^{3}$ Departments of Pediatrics and Medicine, University of Washington, Box 357720, Seattle, WA 98195, USA; ${ }^{4}$ Department of Pathology, University of Washington, Box 357470, Seattle, WA 98195, USA; ${ }^{5}$ Abteilung für Humangenetik, Medizinische Universitäts-Kinderklinik, Inselspital, Bern CH-3010, Switzerland; ${ }^{6}$ Medical Genetic Service, Hospital Dona Estefania, Rua Jacinta Marto, Lisboa 1169-045, Portugal
\end{abstract}

Deletion or mutation of the TRPS1 gene leads to the tricho-rhino-phalangeal syndromes (TRPS). The gene encodes a zinc-finger transcription factor, which contains two regions with basic amino acids LRRRRG (NLS1) and RRRTRKR (NLS2) that resemble potential nuclear localization signals (NLSs). Here, we describe the identification of novel TRPS1 mutations in patients with TRPS type I (TRPS I) and provide, by reconstructing the mutant TRPS1 proteins and subcellular localization studies, evidence that only the RRRTRKR motif functions as a NLS. Two different mutations affect the last arginine residue of this motif. The exchanges of arginine to histidine, found in two unrelated patients with TRPS $I$, as well as the exchange of arginine to cysteine, found in another unrelated patient, prevent the translocation of the mutant TRPS1 to the nucleus when ectopically expressed in COS 7 cells. In contrast, a mutant that lacks the conserved GATA-type zinc-finger domain and most of the LRRRRG motif is able to enter the nucleus. European Journal of Human Genetics (2004) 12, 121-126. doi:10.1038/sj.ejhg.5201094

Published online 22 October 2003

Keywords: TRPS1; nuclear localization signal; novel mutations

\section{Introduction}

After synthesis in the cytoplasm, 'nuclear' transcription factors need to be imported into the nucleus to exert their activity. Although the nuclear pore complex is permeable to molecules as large as $40-60 \mathrm{kDa}$, import into the nucleus is normally an active process. Protein transport is dependent on the presence of one or several nuclear localization signals (NLSs) in the primary structure of the transported protein. ${ }^{1}$ We have recently identified the TRPS1 gene on human chromosome 8q24.1, which

*Correspondence: Dr Hermann-Josef Lüdecke, Institut für Humangenetik, Universitätsklinikum, Hufelandstr. 55, Essen D-45122, Germany. Tel: +49 201 7234555; Fax: + 492017235900 ;

E-mail: hj.luedecke@uni-essen.de

Received 13 June 2003; revised 6 August 2003; accepted 14 August 2003 encodes the 1281 amino acids (aa) zinc-finger transcription factor protein TRPS1 (Figure 1a). It has a calculated molecular mass of $141.6 \mathrm{kDa}$ and contains an unusual combination of nine potential zinc-finger motifs of eight different types. ${ }^{2}$ So far, only the function of the last three zinc-fingers (motifs 7-9) has been determined. Motifs 8 and 9 (1217-1237 and 1245-1267 aa) exhibit a high degree of similarity to the C-terminal double zinc-finger of the IKAROS transcription factor family, ${ }^{2}$ which is required for interaction with self and the other family members HELIOS and AIOLOS, and to mediate the repressive function of the IKAROS proteins. ${ }^{3,4}$ Malik et al have fused the most C-terminal 119 amino acids of the TRPS1 protein, including the IKAROS-like zinc-fingers, to the XGATA4 transcription activator, and found that this part of the TRPS1 protein can override the intrinsic 


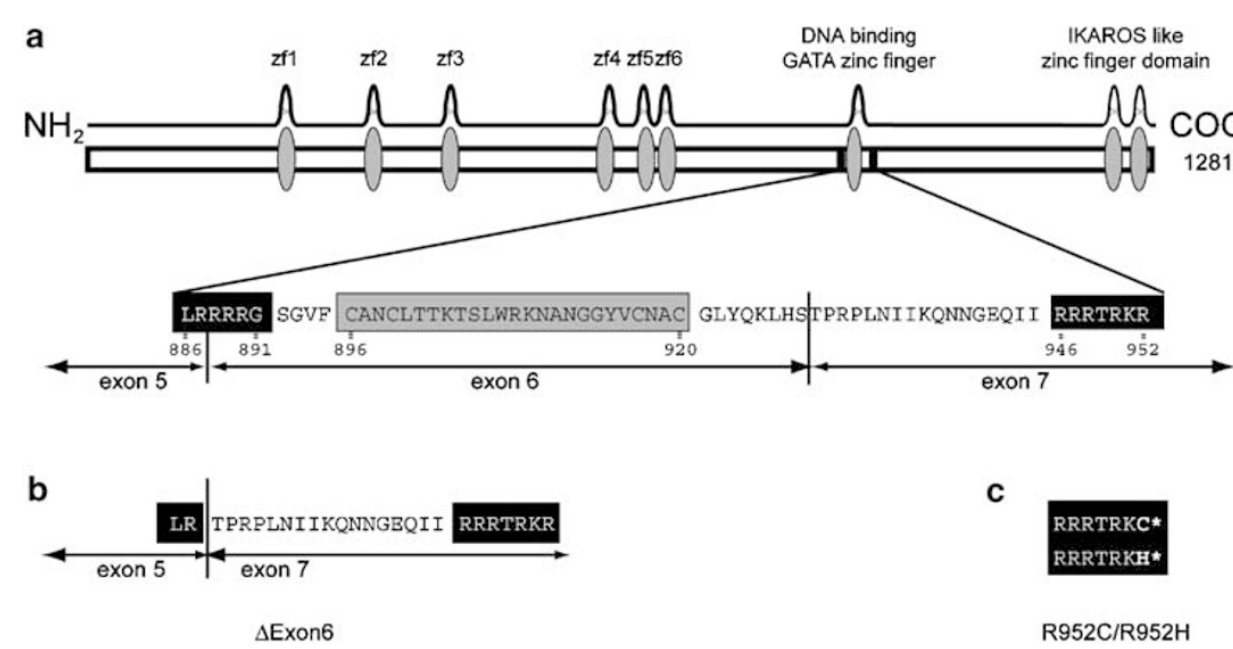

Figure 1 Predicted structure of the wild-type and mutant TRPS1 proteins. (a) The wild-type TRPS1 contains nine potential zinc-finger domains and two potential NLSs. The zinc-fingers are depicted as grey ellipses. The predicted NLSs that flank the GATA zinc-finger are indicated as black boxes. (b, c) depict the mutations and the relevant parts of the $\Delta$ Exon6- and the R952C- and R952H-TRPS1-GFP-constructs, respectively.

activation capacity of XGATA4. The seventh zinc-finger motif (896-920 aa, $\mathrm{C}_{2} \mathrm{C}_{2}$-type) binds to the GATA consensus sequence. An intact GATA zinc-finger is indispensable for the transcription repression activity of TRPS $1 .{ }^{5}$ This GATA zinc-finger is flanked by two basic amino-acid stretches resembling potential monopartite NLSs (NLS1: LRRRRG, 886-891 aa, and NLS2: RRRTRKR, 946-952 aa, Figure 1a). For the GATA-1 and GATA-4 proteins, it has been shown that basic amino-acid residues C-terminal to the DNA-binding zinc-finger are essential for tight DNA binding. ${ }^{6,7}$ Thus, the motif RRRTRKR may also assist in specifying the DNA binding of the GATA zinc-finger. The two potential NLSs and the GATA zinc-finger reside in an 81 aa spanning region, which is $100 \%$ identical in human, mouse, and Xenopus TRPS $1,{ }^{5}$ emphasizing the importance of this region.

Mutations of the TRPS1 gene lead to the tricho-rhinophalangeal syndromes (TRPS) types I (TRPS I; MIM 190350) or III (TRPS III; MIM 190351). The hallmarks of the syndromes are craniofacial and skeletal abnormalities. The patients have sparse scalp hair, a bulbous tip of the nose, a long flat philtrum, and a thin upper vermillion border. The skeletal abnormalities are most probably due to an impaired maturation of chondrocytes in the growth plates of many tubular bones, which causes cone-shaped epiphyses of the phalanges. This leads to varying degrees of brachydactyly and short stature. The TRPS types I and III differ only in two regards, the type of TRPS1 mutations and the severity of the skeletal abnormalities. In a comprehensive study, ${ }^{8}$ we have shown that deletions of one complete TRPS1 allele, as well as nonsense mutations in TRPS1 lead to TRPS type I. On the other hand, missense mutations have only been found in the GATA DNA-binding domain, and are always associated with the more severe TRPS type III. ${ }^{8,9}$ However, a mutation in the splice-donor site of intron 6 resulting in skipping of exon 6 and deletion of the entire GATA zinc-finger coding domain led to a TRPS I phenotype in a female patient (Figure 1b, case 13568 in Lüdecke et $a l)^{8}$.

Here, we describe the first two missense mutations that do not affect the GATA zinc-finger, identified in patients with TRPS I, and the functional analysis of the two predicted NLSs.

\section{Materials and methods Mutation analysis}

DNA samples of a female patient from Switzerland, a fourgeneration family from the USA, and a two-generation family from Portugal were referred to us for TRPS1 mutation screening. We performed mutation analysis by direct sequencing of PCR products from the genomic DNA of the patients, as described previously. ${ }^{2,8}$ This study was approved by the local ethics committee at the Universitätsklinikum Essen.

\section{Construction of a wild-type and three mutant TRPS1-} GFP fusion proteins

We attempted to generate a fusion protein of TRPS1 and green fluorescent protein (GFP).

To enable asymmetric cloning, we generated a PCR product from the plasmid pBS79FL, ${ }^{10}$ which contains the entire open reading frame (ORF) of the human TRPS1 gene, using the primers 5'-EcoRI (5'-CGGAATTCACAGATATGGTCCGG-3', the TRPS1 start codon is typed in bold) and $3^{\prime}$-BamHI (5'-CGGGATCCATACTCTTTAGGTTTTCC-3'), 
and the platinum high-fidelity DNA polymerase (Invitrogen). The PCR product was digested with EcoRI and BamHI, and subcloned into the mammalian expression vector pEGFP-N3 (Clontech), which allows in-frame fusion with enhanced Aequorea victoria GFP under the control of the cytomegalovirus immediate early promoter and contains SV40 polyadenylation signals. This wild-type construct is referred to as WT-TRPS1-GFP.

We constructed mutant TRPS1-GFP fusion proteins containing the amino-acid substitutions R952C (R952CTRPS1-GFP) or R952H (R952H-TRPS1-GFP) with the help of the QuikChange ${ }^{\mathrm{TM}}$ site-directed mutagenesis kit (Stratagene $\left.^{\circledR}\right)$. We used the synthetic complementary oligonucleotides carrying the specific missense mutation $2854 \mathrm{C}>\mathrm{T} \quad\left(5^{\prime}\right.$-CAAGAAAGTGCCTTAACCCAG-3'; ${ }^{\prime}$ ' ${ }^{\prime}-$ CTGGGTTAAGGCACTTTCTTG $\left.-3^{\prime}\right)$ or $2855 \mathrm{G}>\mathrm{A} \quad\left(5^{\prime}-\right.$

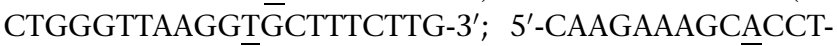
TAACCCAG-3') as primers and the WT-TRPS1-GFP plasmid as a template to generate a mutant amplification product. After removal of the wild-type template DNA by DpnI digestion, the PCR product was used to transform DH5 $\alpha$ cells. To generate the TRPS1 exon 6 deletion construct, we used a modified QuikChange ${ }^{\mathrm{TM}}$-based PCR reaction. Oligonucleotides either complementary to the $3^{\prime}$ end of exon 5 (5'-CCGTAACAGGGACTGGGATTC- $3^{\prime}$ ) or equivalent to the $5^{\prime}$ end of exon 7 (5'-ACTCCCAGGCCTTTAAACCATC-3') and the WT-TRPS1-GFP plasmid were used to generate a cDNA, which lacks the exon 6 coding region. A phosphate residue was attached to the $5^{\prime}$ end of the exon 7 primer to enable blunt-end ligation of the PCR product. The PCR product was DpnI treated, heat inactivated, purified through Microcon ${ }^{\circledR}$ YM-100 centrifugal filter devices (Amicon), blunt-end ligated for $3 \mathrm{~h}$ at room temperature, and used to transform DH5 $\alpha$ cells. All the four fusion constructs were verified by sequencing.

\section{Cell transfections and subcellular localization}

For the subcellular detection of the GFP-fusion proteins, COS 7 cells were cultured in $6 \mathrm{~cm}$ plates $\left(1 \times 10^{5}\right.$ cells/plate $)$ on glass coverslips and transfected $24 \mathrm{~h}$ later in DMEM medium containing $0.5 \%$ fetal calf serum (FCS) with 2$4 \mu \mathrm{g}$ of the respective plasmid by electroporation. At $16 \mathrm{~h}$ after transfection, cells were fed again with fresh medium containing 10\% FCS. After 24-48 h, they were harvested in cold phosphate-buffered saline (PBS), fixed with methanol, again washed with PBS and with IF buffer $(10 \mathrm{~mm}$ Tris $\mathrm{pH}$ $7.5,300 \mathrm{~mm} \mathrm{NaCl}, 0.05 \%(\mathrm{v} / \mathrm{v})$ Tween 20). Cells were then blocked for $30 \mathrm{~min}$ in $1 \%$ bovine serum albumin (BSA) in IF buffer, washed twice with IF buffer and mounted on slides with aquamount (BDh Laboratories). The nuclei were stained by $4^{\prime}, 6^{\prime}$-diamidino-2-phenylindole (DAPI blue). The subcellular localization of the GFP fusion products in at least 100 cells per experiment was analysed by confocal laser scanning microscopy.

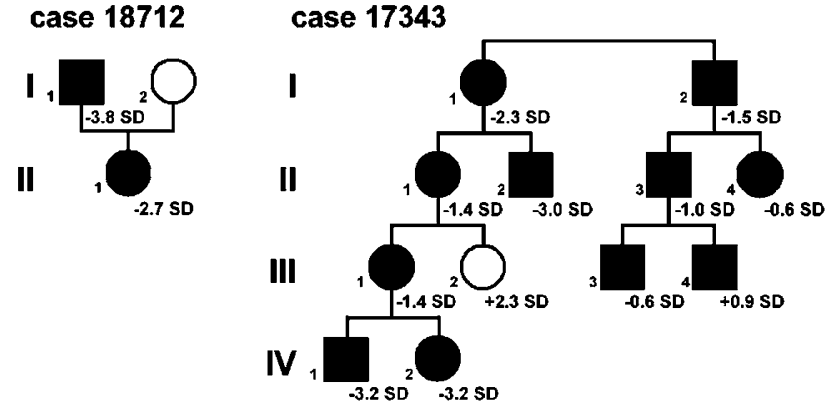

Figure 2 Pedigrees of the familial cases 18712 and 17343. Affected individuals are indicated by filled symbols. Only the family members related by blood are shown in case 17343 . The height is indicated as SD from the mean at the respective age.

\section{Results \\ Identification of a novel missense mutation in the TRPS1 gene}

In our ongoing attempts to augment the spectrum of TRPS1 mutations, we identified two novel mutations that occur in the same CpG dinucleotide in the protein-coding part of exon 7 of the TRPS1 gene. One is a 2854C $>$ T transition found in a father and daughter from Portugal (case 18712, Figure 2). Both are heterozygous $2854 \mathrm{C} / \mathrm{T}$. The other, a $2855 \mathrm{G}>\mathrm{A}$ transition, was found in a female patient from Switzerland (case 15652), and in a fourgeneration family from USA (case 17343, Figure 2). The patient from Switzerland also has a positive family history for TRPS; however, detailed pedigree information or other family members were not available for analysis. To exclude a further mutation, we sequenced in all cases the entire coding region as well as the noncoding exon 2. No additional mutation was found. In case 17343, all individuals related to the index patient III.1 were analysed as well. All affected individuals are heterozygous 2855G/A, but not the only unaffected individual III.2, who is homozygous for wild-type $2855 \mathrm{~A}$.

The $2854 \mathrm{C}>\mathrm{T}$ transition leads to the exchange of arginine to cysteine at position 952 in the TRPS1 protein (R952C, Figure 1c). The 2855G $>$ A transition affects the same amino acid, but leads to an arginine to histidine exchange (R952H, Figure 1c). These are the first two missense mutations in the TRPS1 gene that do not affect the GATA zinc-finger domain.

\section{Anthropometric analysis}

Missense mutations in the GATA zinc-finger domain of TRPS1 lead to severe short stature and brachydactyly, defined as TRPS type III. Whereas the brachydactyly in TRPS I is predominantly due to brachymesophalangy, patients with TRPS III exhibit shortness of nearly all bones of the hands including the metacarpals. In order to 
determine the phenotypic consequence of the first aminoacid changes outside this region, we examined the height and hand radiographs of the patients.

Height measurements were available for all analysed individuals. To be able to compare the height of the patients with our previously published data, ${ }^{8}$ we calculated the standard deviations (SD) from mean height using the values for Western European Caucasians, ${ }^{11}$ which correlate well with the values provided by the National Center for Health Statistics (http://www.cdc.gov/nchs/data/nhanes/ growthcharts/zscore/zstatage.txt). Patient 15652 was $146 \mathrm{~cm}(-3.3 \mathrm{SD})$ at the age of 44 years, and individual III. 1 of case 17343 was $157.5 \mathrm{~cm}(-1.44 \mathrm{SD})$ at the age of 35 years. Interestingly, the height of her affected relatives varied from $-3.23 \mathrm{SD}$ in her children (individuals IV.1, $104 \mathrm{~cm}$ at 6.5 years, and IV.2, $96.5 \mathrm{~cm}$ at 5 years) to +0.89 SD in a male second-degree cousin (individual III.4, $183 \mathrm{~cm}$, adult). Individual I.1 of case 18712 was $152 \mathrm{~cm}$ $(-3.8 \mathrm{SD})$. This height variability is within the range observed earlier in patients with TRPS I. ${ }^{8}$

Original hand X-rays were available from one adult patient of each family. The metacarpophalangeal pattern profiles (MCPPP, Figure 3) of the hand skeletons of the patients were established as described earlier. ${ }^{8}$ All profiles correlate highly significantly with the mean profile for TRPS I, ${ }^{8} r=0.72, P<0.001$ for cases 15652 and 17343; and $r=0.82, P<0.001$ for case 18712 , but are significantly different from the mean TRPS III, ${ }^{8}$ $r=0.27$ (case 15 652), $r=-0.09$ (case 17 343), and $r=0.23$ (case 18712). The MCPPP for case 13568 is also in agreement with a TRPS type I (correlation with TRPS I: $r=0.74 ; P<0.001$; with TRPS III: $r=-0.14)$. This female patient was $156 \mathrm{~cm}(-1.7 \mathrm{SD})$ at the age of 35 and is heterozygous for a splice-donor site mutation of intron $6 .^{8}$
Functional analysis of missense mutations in NLS2 Arginine 952 is the last of the seven amino acids, which represent the predicted NLS 2 (NLS2, RRRTRKR). Although $\mathrm{R} 952 \mathrm{H}$ is an exchange of two basic amino acids, we thought that it might be a true mutation, because we found this particular mutation in two unrelated patients. This was supported by the fact that R952C affects the same residue. To examine the effects of the amino-acid exchanges, we constructed expression plasmids coding for GFP fusion proteins with the wild-type TRPS1 (WT-TRPS1GFP) and the two mutant TRPS1 proteins (R952C-TRPS1GFP and R952H-TRPS1-GFP, see Materials and methods).

The four constructs were transfected separately into COS 7 cells and their subcellular localization was analysed by confocal laser-scanning microscopy (Figure 4), as described in Materials and methods. The WT-TRPS1-GFP fusion protein was evenly distributed throughout the nucleus (Figure $4 \mathrm{a}$ and $\mathrm{a}^{*}$ ). This is different from the intranuclear distribution of the endogenous TRPS1, which is localized in dot-like structures. ${ }^{10}$ In contrast to WT-TRPS1-GFP, both mutant proteins, R952C-TRPS1-GFP (Figure $4 \mathrm{~b}$ and $\mathrm{b}^{*}$ ) and R952H-TRPS1-GFP (Figure $4 c$ and $c^{*}$ ), were exclusively located in the cytoplasm. These results indicate that the predicted NLS2 is required for nuclear localization of TRPS1.

Functional analysis of a TRPS1 splice-site mutation Previously, we had identified a mutation in the splicedonor site of intron 6 , IVS $6+1 \mathrm{G}>\mathrm{T}$, in a patient with TRPS type I. The mutation causes an in-frame skipping of exon 6 . Stability of the mutant mRNA has been shown by RT-PCR. ${ }^{8}$ The resulting truncated TRPS1 protein lacks the entire GATA-type zinc-finger as well as the last four amino acids (RRRG) of the predicted NLS1 (Figure 3b). By in vitro mutagenesis (Material and methods), we generated an

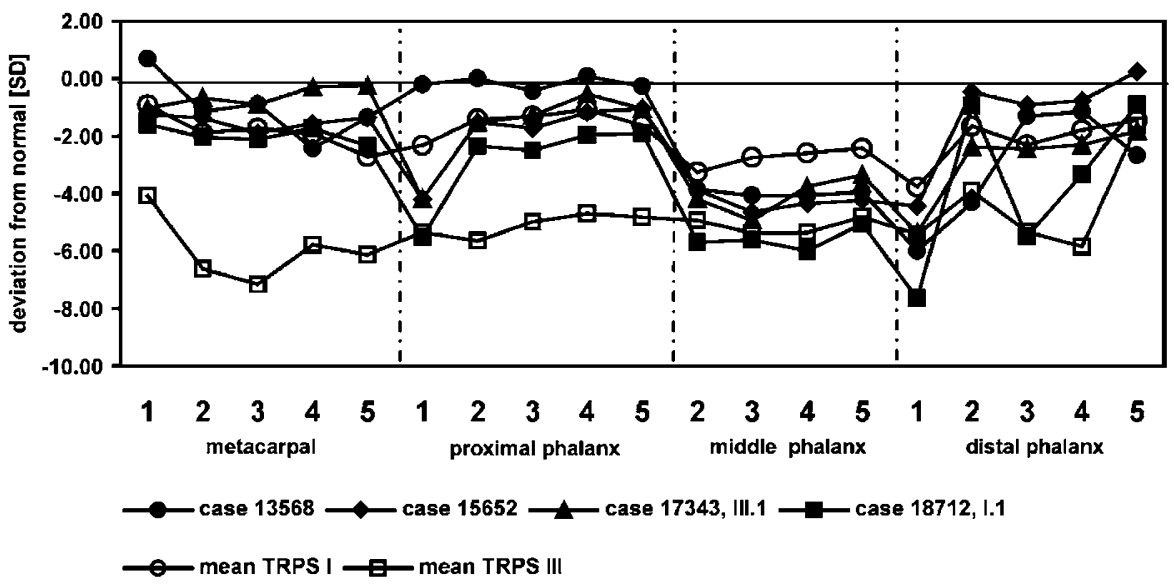

Figure 3 Metacarpophalangeal pattern profiles of one adult patient from each case in comparison to the mean pattern profiles for TRPS types I and III as established previously. ${ }^{8}$ For case 17343 , individual III.1, and for case 18712 , individual I.1 (see Figure 1) were analysed. 

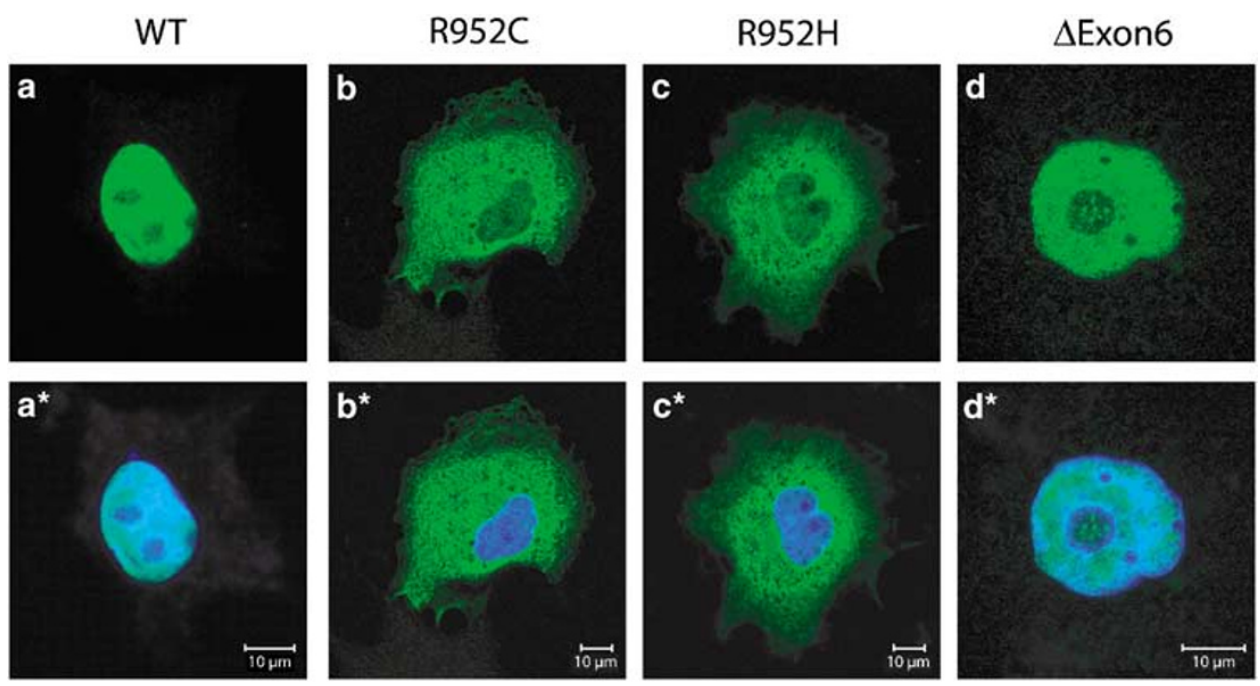

Figure 4 Intracellular localization of wild-type and mutant TRPS1 proteins. We transfected COS 7 cells with wild-type or mutant TRPS1-GFP fusion constructs $(\mathbf{a}-\mathbf{d})$ and analysed the cells by confocal laser-scanning microscopy. We used DAPI blue staining to identify the nuclei $\left(\mathbf{a}^{*}-\mathbf{d}^{*}\right)$. $\left(\mathbf{a}, \mathbf{a}^{*}\right)$ The WT-TRPS1-GFP is exclusively located in the cell nucleus. Both constructs with missense mutations at position 952 in the predicted NLS2 are unable to enter the nucleus. (b, $\left.\mathbf{b}^{\star}\right)$ R952C; $\left(\mathbf{c}, \mathbf{c}^{*}\right)$ R952H; $\left(\mathbf{d}^{*} \mathbf{d}^{*}\right)$ Interestingly, the $\triangle$ Exon6-TRPS1-GFP construct, which lacks the predicted NLS1 but retains the predicted NLS2, enters the nucleus. Note that images are not taken at the same magnification (see size bars).

expression plasmid ( $\triangle$ Exon6-TRPS1-GFP) which codes for the truncated TRPS1 protein. The $\triangle$ Exon6-TRPS1-GFP construct was transfected into COS 7 cells and the subcellular localization was determined as described above. Unexpectedly, $\Delta$ Exon6-TRPS1-GFP was still able to enter the nucleus (Figure $4 \mathrm{~d}$ and $\mathrm{d}^{*}$ ), although it lacks the predicted NLS1. Thus, it is unlikely that the hexapeptide LRRRRG is involved in the transport of TRPS1 into the nucleus.

\section{Discussion}

Although there is no consensus sequence for (NLSs), hexapeptide stretches containing four basic amino acids like arginines, lysines or in some cases even histidines are considered good candidates for NLSs. ${ }^{1}$ The TRPS 1 protein contains two potential NLSs, but they have not been characterized in detail so far. Our functional analyses of naturally occurring TRPS1 mutations now show that the RRRTRKR motif (946-952 aa) is indispensable for translocation of TRPS1 into the nucleus and that a single amino-acid exchange is sufficient to prevent nuclear transfer, whereas disruption of the LRRRRG motif does not prevent nuclear localization. This indicates that only the RRRTRKR motif functions as an NLS in TRPS1.

We have previously shown that TRPS type I is associated with deletions and nonsense mutations of one allele of TRPS $1,{ }^{8}$ which are believed to cause a reduction of the TRPS1 protein concentration in the nucleus, a phenomen- on called haploinsufficiency. In contrast, the more severe TRPS III phenotype was found to be exclusively associated with missense mutations in the GATA zincfinger of TRPS1, and we have hypothesized that this is due to a dominant-negative effect. ${ }^{8}$ The missense mutations R952C and $\mathrm{R} 952 \mathrm{H}$ represent the first missense mutations in TRPS1 which lead to TRPS I. They do not affect the GATA zinc-finger, but prevent the transport of the TRPS1 protein into the nucleus and thus reduce the nuclear TRPS1 concentration. These findings are consistent with the haploinsufficiency model of TRPS I. We noticed that the nuclear distribution pattern of the ectopically expressed WT-TRPS1-GFP (Figure $4 \mathrm{a}$ and $\mathrm{a}^{*}$ ) is different from that of the endogenous protein which is located in dot-like structures. ${ }^{12}$ The homogeneous nuclear distribution of the WT-TRPS1-GFP is probably due to overexpression of this artificial gene. As overexpression does not prevent nuclear entrance of TRPS1, cytoplasmic retention of R952C-TRPS1-GFP and R952H-TRPS1-GFP is due to the mutations and not due to the fusion of TRPS1 and GFP.

TRPS1 has been shown to be a transcriptional repressor of hitherto unknown GATA-regulated genes. Malik et $\mathrm{al}^{5}$ have shown that the transcriptional repression function of TRPS1 is dependent on the presence of both the GATA zincfinger and the IKAROS-like zinc-fingers at the C-terminus. TRPS1 with a missense mutation in the GATA zinc-finger may cause TRPS III by competing with the wild-type TRPS1 for other components in a multimeric transcription control complex. ${ }^{8}$ So far, only two TRPS1 interacting proteins are 
known. ${ }^{12,13}$ The dynein light-chain LC8a interacts with two regions of TRPS1 which flank the GATA zinc-finger, and the RING finger protein RNF4 interacts with a 200 amino-acid region (985-1184 aa) located between the GATA and the IKAROS-like zinc-fingers. Both interactions suppress the transcriptional repression activity of TRPS1. ${ }^{12,13}$ We have previously hypothesized that the splice site mutation in case 13568 , which causes skipping of exon 6 and the deletion of the potential NLS1 and the entire GATA zinc-finger, would prevent the mutant TRPS1 from entering the nucleus. ${ }^{8}$ Here we show that the mutant protein $\triangle$ Exon6-TRPS1-GFP does enter the nucleus. As patient 13568 has a milder form of the disease, complete deletion of the GATA zinc-finger has a milder effect than a missense mutation in it. There are several explanations to account for this surprising result. (I) It is possible that the endogenous mutant protein is misfolded and is subject to the quality-surveillance mechanism and degraded. ${ }^{14}$ This system may not efficiently remove the overexpressed protein. (II) The deleted region may contain a domain through which TRPS1 interacts with other hitherto unknown proteins. If so, a TRPS1 protein without the GATA zinc-finger cannot compete for binding with other proteins and the mutant TRPS1 gene would behave as a null allele in agreement with the TRPS I phenotype of the patient.

Our results emphasize the importance of mutation analyses in patients with TRPS for the identification of the functional domains of the TRPS 1 transcription factor.

\section{Acknowledgements}

We thank the patients and their families for their cooperation, $S$ Tesmann for expert technical assistance, and T Möröy for continuous support. This study was supported by grant Lu483/5-1 from the Deutsche Forschungsgemeinschaft.

\section{References}

1 Boulikas T: Nuclear localization signals (NLS). CRC Crit Rev Euk Gene Expr 1993; 3: 193-227.

2 Momeni P, Glöckner G, Schmidt O et al.: Mutations in a new gene, encoding a zinc-finger protein, cause tricho-rhinophalangeal syndrome type I. Nat Genet 2000; 24: 71-74.

3 Sun L, Liu A, Georgopoulos K: Zinc finger-mediated protein interactions modulate Ikaros activity, a molecular control of lymphocyte development. EMBO J 1996; 15: 5358-5369.

4 Koipally J, Renold A, Kim J, Georgopoulos K: Repression by Ikaros and Aiolos is mediated through histone deacetylase complexes. EMBO J 1999; 18: 3090-3100.

5 Malik TH, Shoichet SA, Latham P, Kroll TG, Peters LL, Shivdasani RA: Transcriptional repression and developmental functions of the atypical vertebrate GATA protein TRPS1. EMBO J 2001; 20: $1715-1725$

6 Omichinski JG, Trainor C, Evans T, Gronenborn AM, Clore GM, Felsenfeld G: A small single-"finger" peptide from the erythroid transcription factor GATA binds specifically to DNA as a zinc or iron complex. Proc Natl Acad Sci USA 1993; 90: 1676-1680.

7 Morrisey EE, Ip HS, Tang Z, Parmacek MS: GATA-4 activates transcription via two novel domains that are conserved within the GATA-4/5/6 subfamily. J Biol Chem 1997; 272: 8515-8524.

8 Lüdecke H-J, Schaper J, Meinecke P et al.: Genotypic and phenotypic spectrum in the tricho-rhino-phalangeal syndrome types I and III. Am J Hum Genet 2001; 68: 81-91.

9 Kobayashi H, Hino M, Shimodahira M et al.: Missense mutation of TRPS1 in a family of tricho-rhino-phalangeal syndrome type III. Am J Med Genet 2002; 107: 26-29.

10 Chang GT, Steenbeek M, Schippers E et al.: Characterization of a zinc-finger protein and its association with apoptosis in prostate cancer cells. J Natl Cancer Inst 2000; 92: 1414-1421.

11 van Wieringen JC, Wafelbakker F, Verbrugge HP, de Haas JH: Growth Diagrams 1965, Netherlands. Groningen, The Netherlands: Wolters-Nordhoff, 1971.

12 Kaiser FJ, Tavassoli K, Van den Bemd G-J et al.: Nuclear interaction of the dynein light chain LC8a with the TRPS1 transcription factor suppresses the transcriptional repression activity of TRPS1. Hum Mol Genet 2003; 12: 1349-1358.

13 Kaiser FJ, Möröy T, Chang GT et al.: The RING finger protein RNF4, a co-regulator of transcription, interacts with the TRPS1 transcription factor. J Biol Chem 2003, [July 28 Epub ahead of print].

14 Clausen T, Southan C, Ehrmann M: The HtrA family of proteases: implications for protein composition and cell fate. Mol Cell 2002; 10: $443-455$ 